has highlighted the existence of a functional barrier in the membrane of polarized neurons during development and underscores the importance of further studies on membrane heterogeneity for better understanding of neuronal functions.

Carlos G. Dotti is at the Cavalieri Ottolenghi Scientific Institute, Universita degli Studi di

Torino, A.O. San Luigi Gonzaga, Regione Gonzole 10, 10043 Orbassano (TO) Italy e-mail:carlos.dotti@unito.it

Mu-ming Poo is at the Division of Neurobiology,

Department of Molecular and Cell Biology,

University of California, Berkeley, Berkeley, CA

94720,USA.e-mail:mpoo@uclink.berkeley.ed

ACKNOWLEDGEMENTS

We thank Vanessa Schubert for making Figure 1.

1. Craig, A. M. \& Banker, G. Ann. Rev. Neurosci. 17 267-310 (1994).

2. Ledesma, M. D. \& Dotti, C. G. 2003. Int. Rev. Cytol. Seminars in Cell Biology, (in the press).

3, Tang, B. L. J. Neurochem. 79, 923-930 (2001).
4. Nakada, C. et al. Nat. Cell Biol. 5, 626-633 (2003).

5. Kobayashi, T. Storrie, B., Simons, K. \& Dotti, C. G. Nature 359, 647-650 (1992).

6. Futerman, A. H., Khanin, R. \& Segel, L. A. Nature 362, 119 (1993)

7. Wincker, B. \& Poo, M-M. Nature 379, 213 (1996).

8. Winckler, B, Forscher, P. \& Mellman, I. Nature 397, 698-701 (1999).

9. Zhou, D. et al. J. Cell Biol. 143, 1295-1304 (1998).

10. Sasaki, S. \& Maruyama, S. J. Neurol. Sci. 110, 114-120 (1992).

11. Ledesma, M. D., Brugger, B., Bunning, C., Wieland, F.T, and Dotti, C. G. EMBO J. 18, 1761-1771 (1999)

12. Silverman, M. A. et al. Proc. Natl Acad. Sci. USA. 98 7051-7057 (2001)

\section{A game of HIV and mouse}

HIV has been a cumbersome object to study. This is not least because in vivo analysis has to be carried out in hosts that support replication of this retrovirus; namely, humans or monkeys. Macaques may breathe a sigh of relief now that Peterlin and colleagues report on page 611 of this issue that another hurdle to replication in murine cells has been overcome.

HIV replication in mouse cells has been studied for some time, and indeed this has resulted in some fundamentally important insights into its biology. Initially, the viral coreceptors CXCR4 (for T-cell tropic HIV strains) and CCR5 (for macrophage-specific strains) were identified as determinants for murine HIV entry along with the human CD4 receptor. Despite resolving the entry problem, viral replication still failed and subsequently the defect was pinpointed to murine CyclinT1 (CycT1), a subunit of the viral Tat transactivator associated factor P-TEFb: expression of human CycT1 in mouse cells was sufficient to rescue Tat-driven viral transcription. However, even expression of human receptor and coreceptor along with CycT1 was not sufficient to support full viral replication in mouse, suggesting that other post-transcriptional blocks must exist.

The single HIV transcript is subject to a complex set of splicing reactions, and a total of 46 RNA species have been reported. Three RNA species stand out as functionally important: the unspliced 9$\mathrm{kb}$ transcript, forming the viral genome and encoding the viral proteins Gag and Pol; a singly spliced 4-kb species encoding the Vif, Vpr, Vpu and Env proteins; and a multiply spliced 2-kb species encoding Tat, Rev and Nef. The regulator of virion gene expression (Rev) is responsible for mediating nuclear export of the 9- and 4$\mathrm{kb}$ species to protect them from splicing, which is an essential step in virus replication. It was noted over a decade ago that only multiply spliced species would accumulate in murine cells and that Rev function was compromised. Indeed, it soon became clear that this defect could be complemented with a factor expressed from human chromosome 11.

Peterlin and colleagues confirm that viral RNA is indeed spliced excessively in murine cells, relative to human cells, with a significant decrease of the 9- and -4kb transcripts. This excessive splicing precludes nuclear export of the longer RNA species by Rev. The authors had previously identified a Rev-associated protein in mouse that is homologous to human splicing-factor-associated protein p32 (hp32). Indeed, hp32 also binds Rev and functions to

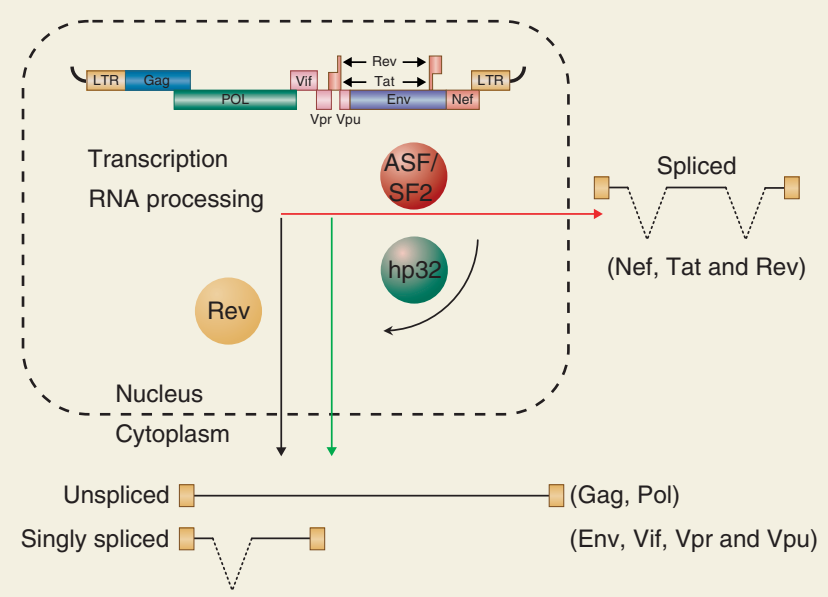

Splicing and transport of HIV transcripts in murine cells. ASF/SF2 commits HIV pre-mRNA to splicing. In murine cells, multiply spliced transcripts are observed in the cytoplasm of infected cells (red arrow). hp32 counteracts effects of ASF/SF2 and facilitates accumulation of genomic and singly spliced viral transcripts transported into the cytoplasm by Rev (green arrow), allowing for assembly of infectious virions.

inhibit HIV splicing in murine cells. Importantly, co-expressed hp32, but not mp32, could rescue Rev activity in murine cells, resulting in increased levels of unspliced HIV genomic RNA. To identify the basis of this difference, murine and human p32 hybrids were tested for inhibition of HIV splicing, narrowing down the region responsible to the amino terminus. Directed mutagenesis of divergent residues identified a single glycine-toaspartate substitution as the culprit. The rescued HIV transcripts gave rise to Gag protein and indeed to fully infectious HIV particles in cell culture supernatants, although levels were not restored entirely to those observed in human cells. hp32 is encoded on chromosome 11, and this data therefore identifies the decade-old Rev-complementing activity. At this time, successful infection of transgenic 'humanized' mice expressing the viral receptors, transcriptional regulator, anti-splicing factor and possibly other interacting proteins remain the keenly awaited proof that HIV can successfully infect this, the favoured model organism. Alternatively, HIV may be 'muranizable' by propagation in replication-competent murine cells. Either way, a mouse susceptible to AIDS would undoubtedly be an invaluable asset to virologists.

BERND PULVERER 